\title{
Impacts of 25-year rotation and tillage management on soil quality in a semi-arid tropical climate
}

\author{
Shuang Zhong ${ }^{1,2^{*}}$, Zhanwu Sheng ${ }^{1,2}$, Lili Zheng ${ }^{1}$, Xiaoyan Zheng ${ }^{1}$, Yang Yang ${ }^{1}$, Dao Xiao ${ }^{1}$, Binling Ai ${ }^{1,2}$, \\ and Huicai Zeng ${ }^{1}$
}

${ }^{1}$ Chinese Academy of Tropical Agricultural Sciences, Haikou Experimental Station, Haikou 570102, China.

${ }^{2}$ Hainan Key Laboratory of Banana Genetic Improvement, Hainan Haikou 570102, China.

"Corresponding author (ongzhish@126.com).

Received: 22 August 2020; Accepted: 10 November 2020; doi:10.4067/S0718-58392021000100003

\begin{abstract}
Soil deterioration, yield decline and soil microbial activity reduction caused by banana (Musa $\times$ paradisiaca L.) monoculture is threatening the sustainability of banana production in China. Therefore, it is necessary to study the benefits of rotation on soil quality. This study aimed to assess the effects of rotation and tillage on soil properties in a banana plantation for $25 \mathrm{yr}$. Treatments consisted of three rotation methods (banana-pineapple, BA; banana-cowpea, BP; banana-rice, BR) and banana monoculture (CK) combined with two tillage intensities (no-tillage, NT; conventional tillage, CT). Soil samples were taken at depth of 0-40 cm in 2019-2020. In comparison with CK, BA and BR increased soil moisture, $\mathrm{pH}$, total organic $\mathrm{C}$ and available $\mathrm{P}$, but decreased soil bulk density. Microbial biomass $\mathrm{C}$ and $\mathrm{N}$ at booting stage were $46.1 \%$ and $39.2 \%$ higher in $\mathrm{BA}$ and $\mathrm{BR}$ than those in $\mathrm{CK}$. Urease, dehydrogenase and $\beta$-glucosidase obtained a mean of $34.1 \%$ increase in $\mathrm{BP}$ and $23.8 \%$ increase in $\mathrm{BR}$ compared with $\mathrm{BA}$. Higher total $\mathrm{N}, \mathrm{NO}_{3}-\mathrm{N}$, available $\mathrm{K}$ and macroaggregate were showed in NT compared with $\mathrm{CT}$, whereas porosity was $24.8 \%$ lower in $\mathrm{NT}$ than in $\mathrm{CT}_{\text {. }} \mathrm{CO}_{2}, \mathrm{~N}_{2} \mathrm{O}$ and $\mathrm{CH}_{4}$ emissions were in average around between one third and two fifth lower in no-tillage compared with conventional tillage. In general, rotations combined with no-tillage led to a positive effect on soil quality, as evidenced by increase of soil moisture, total $\mathrm{N}$, microbial biomass $\mathrm{C}$ and urease and accompanying increase in banana yield. In order to sustain higher productivity, application of rotation and no-tillage is of considerable importance.
\end{abstract}

Key words: Gas emission, microbial biomass, monoculture, rotation, tillage.

\section{INTRODUCTION}

Rotation, no-tillage and crop residue addition greatly improve soil quality, mainly including soil physicochemical and biological properties (Shiwakoti et al., 2019). These practices finally increase crop yield and reduce greenhouse gas emissions (Zuber et al., 2017). For example, rotation potentially improves soil structure and density, decreases acidification and increases nutrient availability (Deuschle et al., 2019). No-tillage involving surface crop residue application has been adopted as a means to promote organic matter storage (He et al., 2020a), which often results in greater soil bacteria biomass and abundance. Meanwhile, no-tillage separates soil organic C (Xavier et al., 2019) and thus to ameliorate $\mathrm{CO}_{2}$ emissions.

Soil microbial properties, such as microbial biomass and enzymes have been suggested as potential indicators for soil quality evaluation because they are involved in soil organic matter decomposition, $C$ sequestration and nutrient availability (Legrand et al., 2018). Furthermore, they are easy to measure (easily adopted for routine laboratory testing), response rapidly to slight changes in less tillage and temporary changes originated by crops rotation. Actually, soil physicochemical and microbial parameters are mutually dependent. Many researchers pointed out that changes in microbial attributes 
with rotation and tillage should provide practical tools to complement physicochemical test (He et al., 2019) and, thus, evaluate the effects of conservation tillage.

With the decrease of per capita arable land area and the increase of cropping intensity, agricultural lands throughout the tropical region are being degraded. Conservation tillage such as integration of no-tillage and residue return into bananabased rotation systems has been proposed as a promising management option to support fruit productivity (Zhong and Zeng, 2020), reduce soil degradation and improve nutrient mineralization in tropical climate. Soil enzymatic activities and microbial biomass as integrative indicators of soil degradation had been assessed in monoculture combined with intensive tillage systems in subtropical climate (Mu et al., 2016), and were shown to be positively affected by conservation tillage practices in short-term studies (Yang et al., 2018). For example, long-term conventional tillage decreased soil enzymes (Bera et al., 2018) and 2 yr tobacco-based rotation increased soil bacterial biomass (Brandan et al., 2017). However, we are uncertain if there will be differences in these soil properties after long-term conservation tillage, especially under tropical fruit plantations. Therefore, the current study measured selected soil quality characters such as soil microbial biomass $\mathrm{C}$ and $\mathrm{N}$, enzymatic activities and greenhouse gas emission involved in $\mathrm{C}$ and $\mathrm{N}$ cycling after $25 \mathrm{yr}$ in bananapineapple, cowpea and rice rotation compared to a 25 yr banana monoculture under no- and conventional tillage.

\section{MATERIALS AND METHODS}

\section{Site descriptions}

The experiment was carried out on the Ledong Experimental Station (18 36'39.2” N, 108 47'54.9” E), Chinese Academy of Tropical Agricultural Sciences since 1994. Climate of the region is tropical monsoon. Average annual temperature is $25.8^{\circ} \mathrm{C}$ and average annual precipitation is $2065 \mathrm{~mm}$. The test soil is aquic Cambisol (13.6\% clay, $23.1 \%$ silt and $63.3 \%$ sand) according to the USDA texture classification; $7.12 \mathrm{~g} \mathrm{~kg}^{-1}$ total organic $\mathrm{C}, 0.76 \mathrm{~g} \mathrm{~kg}^{-1}$ total N, $0.59 \mathrm{~g} \mathrm{~kg}^{-1}$ total $\mathrm{P}$, $1.21 \mathrm{~g} \mathrm{~kg}^{-1}$ total $\mathrm{K}$ and $\mathrm{pH}$ 6.53. Soil moisture, bulk density, porosity, water-stable aggregates (macroaggregate WSA $\mathrm{W}_{1}$, microaggregate $\mathrm{WSA}_{2}$ ) were evaluated.

The experiment was a split-plot design with four replicates. Rotation management was the main plot and tillage system was the split-plot factor. Details of the treatments are showed in Table 1. The text field was divided into 32 plots and size of each plot was $80(10 \times 8 \mathrm{~m}) \mathrm{m}^{2}$. Rotation treatments were banana (Musa $\times$ paradisiaca $\mathrm{L}$.)-pineapple (Ananas comosus [L.] Merr. var. comosus) rotation (BA), banana-cowpea (Vigna unguiculata [L.] Walp.) rotation (BP), banana-rice (Orysa sativa $\mathrm{L}$.) rotation (BR) and banana monoculture (CK). Tillage treatments were no-tillage (NT) and conventional tillage (CT). Each year, cow biochar compost (14.4 tha ${ }^{-1}$ ), with $53.3 \%$ water content, $145 \mathrm{~g} \mathrm{C} \mathrm{kg}^{-1}, 3.2 \mathrm{~g} \mathrm{~N} \mathrm{~kg}^{-1}, 2.5 \mathrm{~g} \mathrm{P}_{2} \mathrm{O}_{5} \mathrm{~kg}^{-1}$, $1.6 \mathrm{~g} \mathrm{~K}_{2} \mathrm{O} \mathrm{kg}^{-1}$, were applied as basal fertilizer. Urea, superphosphate and sulfate were applied as additional fertilizer at $129 \mathrm{~kg} \mathrm{~N} \mathrm{ha}^{-1}, 68 \mathrm{~kg} \mathrm{Pha}^{-1}$ and $292 \mathrm{~kg} \mathrm{~K} \mathrm{ha}^{-1}$. CT plots were moldboard ploughed to $40 \mathrm{~cm}$ depth every year. NT plots were undisturbed, except when the crop was planted using a NT planter (2BQ-6, Kinze, Williamsburg, Iowa, USA). Residues were incorporated into soil in $\mathrm{CT}$ and covered soil surface in NT after harvest.

\section{Soil sampling}

Soil samples were taken from 0-40 cm depth at seedling stage (19 September 2019), jointing stage (18 December 2019), booting stage (15 March 2020) and ripening stage (17 May 2020) within the rows of banana for physicochemical properties, microbial biomass and enzymes analysis. Each sample was a composite comprising five random cores $(2.5 \mathrm{~cm}$ diameter). The fresh samples were sieved through a $2 \mathrm{~mm}$ mesh and stored at $4{ }^{\circ} \mathrm{C}$ before subsequent analysis. Results were based on oven-dried weight of the soil.

Table 1. Description and site history of different treatments in the study area of a long-term crop rotation experiment.

\begin{tabular}{lll}
\hline Treatment & \multicolumn{1}{c}{ Crops } & \multicolumn{1}{c}{ Planting year } \\
\hline $\begin{array}{l}\text { Monoculture (CK) } \\
\text { Rotation 1 (BA) }\end{array}$ & Banana & June 1995-May 2020 \\
& Banana and pineapple & $\begin{array}{l}\text { Pineapple: June 2000-May 2005 and June 2010-May 2015 } \\
\text { Banana: June 1995-May 2000, June 2005-May 2010, and June 2015-May 2020 } \\
\text { Rotation 2 (BP) }\end{array}$ \\
Botation 3 (BR) & Cowpea: June 2000-May 2005 and June 2010-May 2015 \\
& & $\begin{array}{l}\text { Banana: June 1995-May 2000, June 2005-May 2010, and June 2015-May 2020 } \\
\text { Rice: June 2000-May 2005 and June 2010-May 2015 }\end{array}$ \\
\hline
\end{tabular}

In each rotation and monoculture plot, a no-tillage and a conventional tillage treatment were applied. 
Soil physical and chemical properties were measured according to Kubar et al. (2018) and Hulugalle et al. (2007), respectively. Soils were weighed wet, oven-dried at $105^{\circ} \mathrm{C}$ for $8 \mathrm{~h}$, then weighed again to calculate soil moisture, bulk density and porosity. Soil water-stable aggregates were determined by the wet sieving method. Soil $\mathrm{pH}$ was measured in 1:4 soil:water suspension with glass electrode. Total $\mathrm{N}, \mathrm{NH}_{4}-\mathrm{N}$ and $\mathrm{NO}_{3}-\mathrm{N}$ were determined using micro-Kjeldahl method. Total organic $\mathrm{C}$ was analyzed by dry combustion, using a total organic $\mathrm{C}$ analyzer (TOC 5000 total C analyzer; Shimadzu Corporation, Kyoto, Japan). After nitric-perchloric acid digestion, available $\mathrm{P}$ was determined by molybdenum-blue complex method; available $\mathrm{K}$ was analyzed by flame atomic absorption spectrometry; exchangeable $\mathrm{Ca}$ and exchangeable $\mathrm{Mg}$ were determined by atomic absorption spectrophotometry.

Urease, acid phosphatase, and dehydrogenase were determined based on the method of Tabatabai (1994). For urease, $5 \mathrm{~g}$ soil were incubated with $10 \mathrm{~mL} 10 \%$ urea solution for $24 \mathrm{~h}$ at $\mathrm{pH} 7.0$ at $37^{\circ} \mathrm{C}$; for acid phosphatase, $1 \mathrm{~g}$ soil was incubated with the $p$-nitrophenyl phosphate substrate for $1 \mathrm{~h}$ at $\mathrm{pH} 6.5$ at $37^{\circ} \mathrm{C}$; for dehydrogenase, $6 \mathrm{~g}$ soil were incubated with 5 $\mathrm{mL} 3 \%$ triphenyltetrazolium chloride for $24 \mathrm{~h}$ at pH 7.5 at $37^{\circ} \mathrm{C}$. Invertase, $\beta$-glucosidase and catalase were estimated according to the method of Notaro et al. (2018). For invertase, $5 \mathrm{~g}$ soil were incubated with $15 \mathrm{~mL} 8 \%$ sucrose solution for $24 \mathrm{~h}$ at $\mathrm{pH} 5.5$ at $37^{\circ} \mathrm{C}$; for $\beta$-glucosidase $1 \mathrm{~g}$ soil was incubated; and for catalase, $2 \mathrm{~g}$ soil were incubated with $5 \mathrm{~mL}$ $0.3 \% \mathrm{H}_{2} \mathrm{O}_{2}$ for $30 \mathrm{~min}$ at $\mathrm{pH} 7.0$ at $30^{\circ} \mathrm{C}$. When their interactions were significant, individual comparisons were based on $p$-nitrophenyl- $\beta$-D-glucopyranoside substrate for $1 \mathrm{~h}$ at $\mathrm{pH} 6.0$ at $37^{\circ} \mathrm{C}$. Enzymes were determined in fresh soil and based on the oven-dried soil weight.

Soil microbial biomass was measured by the chloroform fumigation-extraction method (Vance et al., 1987). Greenhouse gas emissions were measured every $40 \mathrm{~d}$ after crops transplanting; $\mathrm{CO}_{2}, \mathrm{NO}_{2}$ and $\mathrm{CH}_{4}$ emissions were measured by nonsteady state flow-through chambers (da Vitória et al., 2019). The chamber $(0.5 \mathrm{~m} \times 0.5 \mathrm{~m} \times 2.0 \mathrm{~m})$ with water groove on the top edge, was randomly installed into each plot to a $20 \mathrm{~cm}$ soil depth between two rows of bananas. A rubber stopper with a 3-way stopcock was placed in the wall of each chamber to take gas samples. Samples were analyzed by gas chromatography (HP-6890 gas chromatograph) equipped with a headspace autoanalyzer (HT3) (Agilent Technologies, Barcelona, Spain). Banana yield $\left(\mathrm{t} \mathrm{ha}^{-1}\right)$ was estimated based on bunch weights, mat spacing, and average crop cycle duration (i.e., time between two subsequent harvests from the same mat) as used by Wairegi and van Asten (2010) in the same plots.

\section{Statistical analysis}

The genera linear model (GLM) for split-plot design was used to test the overall effect of rotation, tillage and sampling stage on soil microbial qualities and physicochemical properties. Separate one-way ANOVA was used to test the effects of rotation and tillage on soil microbial properties and environmental parameters at each sampling stage. The mean comparisons were based on an independent-samples $\mathrm{T}$ test. Linear correlation was used to characterize the relationship between physicochemical-microbiological parameters and greenhouse gas. All statistical analyses were performed by SPSS statistical software (SPSS Inc., Chicago, Illinois, USA). Difference at $\mathrm{P}<0.05$ level was considered significant.

\section{RESULTS}

\section{Soil physicochemical property}

Compared with monoculture, rotations significantly increased $(\mathrm{P}<0.05$, Figure 1 and Table 2$)$ almost all of the soil physicochemical properties, but decreased $(\mathrm{P}<0.01)$ bulk density at the $3^{\text {rd }}$ and $4^{\text {th }}$ stage. For rotation treatments, BA had the lowest total soil organic $\mathrm{C}$ and available $\mathrm{P}$; $\mathrm{BP}$ had the highest porosity, available $\mathrm{K}$ and exchangeable $\mathrm{Ca}$; $\mathrm{BR}$ had the highest $\mathrm{NH}_{4}-\mathrm{N}$ and exchangeable $\mathrm{Mg}$. Soil moisture, $\mathrm{pH}$, total soil organic $\mathrm{C}$, total $\mathrm{N}, \mathrm{NO}_{3}-\mathrm{N}$ and exchangeable $\mathrm{Mg}$ were on average $39.4 \%$ higher in no-tillage compared with conventional tillage.

The amounts of macroaggregates $\left(\mathrm{W}_{1}-\mathrm{W}_{4}\right)$ were significantly higher than that of microaggregates $\left(\mathrm{W}_{5}\right)$ in all treatments at the $2^{\text {nd }}$ and $3^{\text {rd }}$ stage $(\mathrm{P}<0.01)$. The amounts of $\mathrm{W}_{2}, \mathrm{~W}_{3}$ and $\mathrm{W}_{4}$ followed the range of $\mathrm{CK}<\mathrm{BA}<\mathrm{BP}<\mathrm{BR}$. Higher $\mathrm{W}_{5}$ were found in NT than in $\mathrm{CT}(\mathrm{P}<0.05$, Table 3$)$. 
Figure 1. Soil chemical properties in rotation and tillage treatments at four sampling stages in a long-term crop rotation experiment.
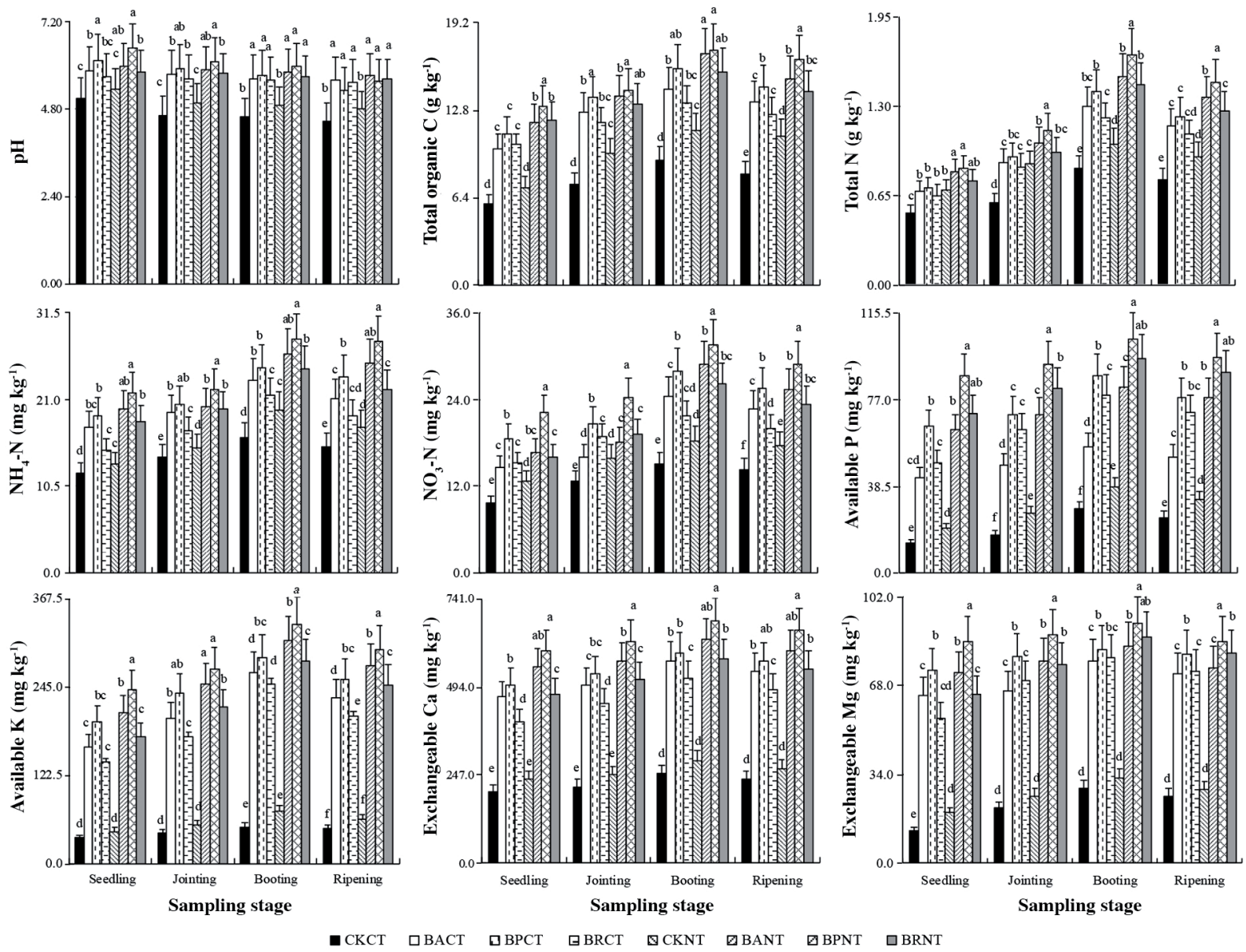

Error bars indicate coefficient of variation. Different letters mean significant difference according to Duncan's multiple range test $(\mathrm{P}<0.05)$. BA: Banana-pineapple rotation; BP: banana-cowpea rotation; BR: banana-rice rotation; CK: banana monoculture; NT: no-tillage; CT: conventional tillage.

Table 2. Soil physical properties and banana yield in rotation and tillage treatments at four sampling stages in a longterm crop rotation experiment.

\begin{tabular}{|c|c|c|c|c|c|c|c|c|c|}
\hline \multirow[b]{2}{*}{ Indicators } & \multirow[b]{2}{*}{ Sampling } & \multicolumn{2}{|c|}{ CK } & \multicolumn{2}{|c|}{ BA } & \multicolumn{2}{|c|}{$\mathrm{BP}$} & \multicolumn{2}{|c|}{$\mathrm{BR}$} \\
\hline & & $\mathrm{CT}$ & NT & $\mathrm{CT}$ & NT & $\mathrm{CT}$ & NT & $\mathrm{CT}$ & NT \\
\hline \multirow{4}{*}{ Soil moisture, $\%$} & $1^{\text {st }}$ & 17.2(1.6)d & $19.2(1.7) \mathrm{d}$ & $22.8(2.1) \mathrm{c}$ & $24.9(2.2) \mathrm{c}$ & $26.8(2.4) \mathrm{b}$ & $29.5(2.6) \mathrm{a}$ & 28.1(2.5)ab & $30.4(2.7) \mathrm{a}$ \\
\hline & $2^{\text {nd }}$ & $19.1(1.8) \mathrm{c}$ & $21.9(1.9) \mathrm{c}$ & $24.3(2.2) \mathrm{b}$ & $26.5(2.4) b$ & $30.7(2.7) \mathrm{a}$ & $31.6(2.9) \mathrm{a}$ & $31.8(2.8) \mathrm{a}$ & $33.1(3.1) \mathrm{a}$ \\
\hline & $3^{\text {rd }}$ & $23.4(2.2) \mathrm{c}$ & $26.8(2.4) \mathrm{b}$ & $28.4(2.6) \mathrm{b}$ & $30.6(2.8) \mathrm{ab}$ & $32.9(3.0) \mathrm{a}$ & $33.4(3.1) \mathrm{a}$ & $34.6(3.2) \mathrm{a}$ & $35.9(3.3) \mathrm{a}$ \\
\hline & $4^{\text {th }}$ & $21.7(2.0) \mathrm{d}$ & $24.6(2.2) \mathrm{c}$ & $27.8(2.5) \mathrm{c}$ & $28.8(2.6) b c$ & $31.5(2.8) \mathrm{b}$ & $32.2(3.0) \mathrm{ab}$ & $33.3(3.1) \mathrm{a}$ & $34.7(3.2) \mathrm{a}$ \\
\hline \multirow{4}{*}{$\begin{array}{l}\text { Bulk density, } \\
\mathrm{g} \mathrm{cm}^{-3}\end{array}$} & $1^{\mathrm{st}}$ & $1.37(0.13) \mathrm{b}$ & $1.51(0.14) \mathrm{a}$ & $1.28(0.12) \mathrm{c}$ & $1.41(0.13) \mathrm{a}$ & $1.32(0.13) \mathrm{b}$ & $1.46(0.14) \mathrm{a}$ & $1.25(0.12) \mathrm{c}$ & $1.39(0.13) \mathrm{ab}$ \\
\hline & $2^{\text {nd }}$ & $0.94(0.09) \mathrm{b}$ & $1.34(0.13) \mathrm{a}$ & $0.88(0.08) \mathrm{c}$ & $0.93(0.09) \mathrm{b}$ & $0.91(0.09) \mathrm{bc}$ & $1.06(0.09) \mathrm{b}$ & $0.82(0.07) \mathrm{c}$ & $0.85(0.07) \mathrm{c}$ \\
\hline & $3^{\text {rd }}$ & $1.09(0.11) \mathrm{a}$ & $1.17(0.12) \mathrm{a}$ & $1.00(0.09) \mathrm{b}$ & $1.02(0.10) \mathrm{b}$ & $0.88(0.08) \mathrm{c}$ & $0.92(0.09) \mathrm{c}$ & $0.93(0.08) \mathrm{b}$ & $0.90(0.08) \mathrm{c}$ \\
\hline & $4^{\text {th }}$ & $1.13(0.12) \mathrm{a}$ & $1.25(0.13) \mathrm{a}$ & $0.99(0.09) \mathrm{c}$ & $1.07(0.11) \mathrm{b}$ & $1.03(0.10) \mathrm{b}$ & $1.11(0.11) \mathrm{a}$ & $0.95(0.09) \mathrm{c}$ & $0.98(0.09) \mathrm{c}$ \\
\hline \multirow[t]{4}{*}{ Porosity, $\%$} & $1^{\text {st }}$ & $44.4(4.2) \mathrm{c}$ & $40.2(3.8) \mathrm{d}$ & $48.3(4.5) b$ & 46.7(4.4)bc & $55.5(5.3) \mathrm{a}$ & $51.0(4.8) \mathrm{ab}$ & $53.3(4.8) \mathrm{a}$ & $47.9(4.5) \mathrm{b}$ \\
\hline & $2^{\text {nd }}$ & $54.3(5.3) \mathrm{c}$ & $52.6(5.1) \mathrm{c}$ & 61.1(5.9)b & $62.3(5.8) \mathrm{ab}$ & $67.0(6.3) \mathrm{a}$ & $65.9(6.2) \mathrm{a}$ & 63.7(6.1)a & $60.0(6.0) \mathrm{b}$ \\
\hline & $3^{\text {rd }}$ & $57.8(5.5) \mathrm{b}$ & $56.3(5.2) \mathrm{c}$ & $61.8(5.8) \mathrm{a}$ & $58.7(5.6) \mathrm{c}$ & $63.6(6.1) \mathrm{a}$ & $62.5(6.0) \mathrm{a}$ & $60.4(6.0) \mathrm{a}$ & $59.5(5.9) \mathrm{ab}$ \\
\hline & $4^{\text {th }}$ & $51.7(5.1) \mathrm{c}$ & $50.5(4.9) \mathrm{c}$ & 58.4(5.7)a & $53.0(5.1) \mathrm{b}$ & 60.4(5.9)a & $57.4(5.8) \mathrm{a}$ & $56.6(5.7) \mathrm{a}$ & $53.8(5.6) \mathrm{b}$ \\
\hline Yield, tha $^{-1}$ & & 18.1(3.2)d & $24.2(3.7) \mathrm{d}$ & $35.9(4.0) \mathrm{c}$ & $40.4(4.2) \mathrm{c}$ & $44.9(4.7) \mathrm{b}$ & $50.8(5.0) \mathrm{a}$ & $41.7(4.5) \mathrm{bc}$ & $48.6(4.6) \mathrm{b}$ \\
\hline
\end{tabular}

Values represented in the table are means across replicates of each plot. Coefficients of variation are in parentheses.

CK: Banana monoculture; BA: banana-pineapple rotation; BP: banana-cowpea rotation; BR: banana-rice rotation; CT: conventional tillage; NT: no-tillage. 
Table 3. Size classes of soil water-stable aggregates (WSA) in rotation and tillage treatments at four sampling stages in a long-term crop rotation experiment.

\begin{tabular}{|c|c|c|c|c|c|c|c|c|c|}
\hline \multirow[b]{2}{*}{ WSA $\left(\mathrm{g} \mathrm{kg}^{-1}\right)$} & \multirow[b]{2}{*}{ Sampling } & \multicolumn{2}{|c|}{$\mathrm{CK}$} & \multicolumn{2}{|c|}{$\mathrm{BA}$} & \multicolumn{2}{|c|}{$\mathrm{BP}$} & \multicolumn{2}{|c|}{ BR } \\
\hline & & $\mathrm{CT}$ & NT & $\mathrm{CT}$ & NT & $\mathrm{CT}$ & NT & $\mathrm{CT}$ & NT \\
\hline \multirow[t]{4}{*}{$>5 \mathrm{~mm}\left(\mathrm{~W}_{1}\right)$} & $1^{\mathrm{st}}$ & $78.9(8.5) \mathrm{d}$ & 107.4(11.6)d & $122.0(13.1) \mathrm{c}$ & $150.4(16.2) \mathrm{b}$ & $143.9(15.5) \mathrm{bc}$ & 156.7(16.7)ab & $134.8(14.5) \mathrm{c}$ & 160.2(17.2)a \\
\hline & $2^{\text {nd }}$ & $90.4(9.7) \mathrm{e}$ & $123.5(13.3) \mathrm{d}$ & $138.9(14.9) \mathrm{d}$ & $170.6(18.3) \mathrm{b}$ & $158.1(17.0) \mathrm{c}$ & 175.3(18.9)b & $165.7(17.8) b c$ & $189.9(20.4) \mathrm{a}$ \\
\hline & $3^{\text {rd }}$ & $132.7(14.3) \mathrm{d}$ & $151.3(16.4) \mathrm{c}$ & $166.7(17.9) \mathrm{c}$ & $198.8(21.5) \mathrm{b}$ & 189.6(20.4)b & 219.6(23.7)ab & 210.4(22.6)b & $223.5(24.0) \mathrm{a}$ \\
\hline & $4^{\text {th }}$ & 119.1(12.8)d & $140.2(15.1) \mathrm{c}$ & $149.8(16.2) \mathrm{c}$ & 182.9(19.7)b & 173.4(18.6)b & 200.5(21.7)a & 184.5(19.8)b & 215.6(23.2)a \\
\hline \multirow[t]{4}{*}{ 5-3 mm $\left(\mathrm{W}_{2}\right)$} & $1^{\mathrm{st}}$ & 163.6(17.7)e & $186.1(20.0) \mathrm{d}$ & $219.5(23.6) \mathrm{c}$ & $240.8(25.9) \mathrm{b}$ & $230.7(24.8) b$ & $272.9(29.3) \mathrm{a}$ & 259.9( & 283.4 \\
\hline & $2^{\text {nd }} \quad 1$ & $178.4(19.2) \mathrm{d}$ & 194.7(20.9)d & $231.4(24.8) \mathrm{c}$ & $262.7(28.3) \mathrm{b}$ & $250.9(27.0) \mathrm{bc}$ & $283.7(30.5) \mathrm{a}$ & 271.2( & $299.8(32.2) \mathrm{a}$ \\
\hline & $3^{\text {rd }}$ & 212.7(22.9)e & $226.6(24.4) \mathrm{d}$ & $270.0(29.0) \mathrm{c}$ & $301.9(32.5) \mathrm{b}$ & $278.8(30.0) \mathrm{c}$ & $317.5(34.1) \mathrm{a}$ & $315.7(33.8) \mathrm{a}$ & $324.6(34.9) \mathrm{a}$ \\
\hline & $4^{\text {th }}$ & 201.8(21.7)d & $207.5(22.3) \mathrm{d}$ & $254.6(27.4) \mathrm{c}$ & $273.2(29.8) \mathrm{c}$ & $266.2(28.6) \mathrm{c}$ & $300.3(32.4) \mathrm{ab}$ & $291.0(31.3) \mathrm{b}$ & $307.8(33.1) \mathrm{a}$ \\
\hline \multirow[t]{4}{*}{ 3-1 mm $\left(\mathrm{W}_{3}\right)$} & $1^{\mathrm{st}}$ & 214.5(23.1)d & 229.4(24.7)c & $236.9(25.5) \mathrm{c}$ & $257.2(27.6) \mathrm{c}$ & 289.3(31.1)b & $314.6(33.8) \mathrm{a}$ & $292.5(31.5) b$ & 318.7 \\
\hline & $2^{\text {nd }} \quad 2$ & $221.1(23.8) \mathrm{d}$ & 248.3(26.6)c & $244.5(26.3) \mathrm{c}$ & $280.9(30.2) b c$ & $302.6(32.5) \mathrm{b}$ & $330.2(3$ & 305.4( & 341.9 \\
\hline & $3^{\text {rd }}$ & $255.4(27.5) \mathrm{e}$ & $276.8(29.9) \mathrm{d}$ & $287.8(30.9) \mathrm{c}$ & $310.5(33.4) \mathrm{c}$ & $327.1(35.3) \mathrm{bc}$ & $360.3(38.7) \mathrm{ab}$ & $340.1(36.6) \mathrm{b}$ & 374.1(40.2)a \\
\hline & $4^{\text {th }}$ & $233.2(25.2) \mathrm{d}$ & $261.9(28.2) \mathrm{c}$ & $269.7(29.0) \mathrm{c}$ & 298.7(32.1)bc & $316.3(34.2) \mathrm{b}$ & $349.5(37.6) \mathrm{a}$ & $329.6(35.4) \mathrm{b}$ & $353.4(38.0) \mathrm{a}$ \\
\hline \multirow[t]{4}{*}{$1-0.25 \mathrm{~mm}\left(\mathrm{~W}_{4}\right)$} & $1^{\text {st }}$ & 320.6(34.6)e & 369.5(39.7)d & $398.4(42.8) \mathrm{cd}$ & d 407.3(43.8)c & 435.5(46.9)bc & $478.6(51$ & $460.0(49.5) \mathrm{b}$ & $500.8(53.8) \mathrm{a}$ \\
\hline & $2^{\text {nd }} \quad 3$ & $335.9(36.3) \mathrm{e}$ & $384.2(41.3) \mathrm{d}$ & $400.6(43.1) \mathrm{c}$ & $422.2(45.4) \mathrm{c}$ & $453.2(48.6) \mathrm{b}$ & $502.7(54.1) \mathrm{a}$ & $477.5(51.2) \mathrm{b}$ & 516.7(55.6)a \\
\hline & $3^{\text {rd }}$ & $357.7(38.4) \mathrm{e}$ & $421.7(45.3) \mathrm{d}$ & $440.3(47.3) \mathrm{d}$ & $457.8(49.2) \mathrm{cd}$ & $484.4(52.1) \mathrm{c}$ & $539.4(58.0) \mathrm{ab}$ & $514.8(55.4) \mathrm{b}$ & 553.5(59.5)a \\
\hline & $4^{\text {th }}$ & $344.8(37.1) \mathrm{e}$ & $395.6(42.5) \mathrm{d}$ & $428.9(46.2) \mathrm{c}$ & $436.1(46.9) \mathrm{c}$ & $469.7(50.5) b c$ & 523.2(56.5)ab & $495.4(53.3) \mathrm{b}$ & $540.9(58.4) \mathrm{a}$ \\
\hline \multirow[t]{4}{*}{$<0.25 \mathrm{~mm}\left(\mathrm{~W}_{5}\right)$} & $1^{\mathrm{st}}$ & $362.3(39.0) \mathrm{e}$ & 390.1(41.9)d & $430.2(46.4) \mathrm{c}$ & $451.9(48.6) \mathrm{c}$ & $486.8(52.3) \mathrm{ab}$ & $520.5(56.0) \mathrm{a}$ & $479.3(51.6) \mathrm{b}$ & 525.6(56.5)a \\
\hline & $2^{\text {nd }}$ & $388.5(41.8) \mathrm{e}$ & $410.8(44.2) \mathrm{d}$ & $439.5(47.2) \mathrm{c}$ & $465.6(50.1) b c$ & $499.9(53.8) \mathrm{b}$ & 531.1(57.3)a & $490.9(52.8) \mathrm{b}$ & \\
\hline & $3^{\text {rd }}$ & 416.5(44.9)d & $444.4(47.8) \mathrm{d}$ & $472.1(50.7) \mathrm{c}$ & $506.7(54.5) \mathrm{bc}$ & $536.6(57.7) \mathrm{b}$ & $559.8(60.2) \mathrm{a}$ & $521.7(56.1) \mathrm{b}$ & $578.4(62.2) \mathrm{a}$ \\
\hline & $4^{\text {th }}$ & $404.0(43.4) \mathrm{e}$ & $426.3(45.8) \mathrm{d}$ & $457.7(49.6) \mathrm{c}$ & $480.4(51.7) b c$ & $511.5(55.0) \mathrm{b}$ & $546.9(58.8) \mathrm{ab}$ & $507.2(54.5) \mathrm{b}$ & 567.3(59.9)a \\
\hline
\end{tabular}

Values represented in the table are means across replicates of each plot. Coefficients of variation are in parentheses.

CK: Banana monoculture; BA: banana-pineapple rotation; BP: banana-cowpea rotation; BR: banana-rice rotation; CT: conventional tillage; NT: no-tillage.

\section{Soil biochemical property}

Microbial biomass C (MBC), urease (UA), $\beta$-glucosidase (GA) and dehydrogenase (DHA) were significantly higher $(\mathrm{P}<0.01$, Table 4) in the treatments NT than in CK-CT. Microbial biomass N $(\mathrm{MBN})$, invertase (IA), DHA and acid phosphatase (APA) were on average $42.1 \%, 47.9 \%$ and $36.7 \%$ higher in BA, BP and BR compared with CK, respectively. Average MBN and catalase (CA) were $28.1 \%$ and $39.6 \%$ higher in NT compared with CT (Figure 2). At the booting stage, MBC, MBN and DHA were much higher than those at other stages.

\section{Greenhouse gas emissions}

Peaks of $\mathrm{N}_{2} \mathrm{O}(\mathrm{NE})$ and $\mathrm{CO}_{2}(\mathrm{CE})$ emissions in rotation and tillage were detected at 200 and $240 \mathrm{~d}$ followed by 160,280 and $320 \mathrm{~d}$, while 40 and $80 \mathrm{~d}$ had the lowest emissions (Figure 3). Average CE ranged from 11.8 to $31.9 \mu \mathrm{mol} \mathrm{m}^{-2} \mathrm{~s}^{-1}$, with the following order: $\mathrm{BP}<\mathrm{BR}<\mathrm{BA}<\mathrm{CK}$; average $\mathrm{NE}$ ranged from 14.9 to $38.1 \mu \mathrm{mol} \mathrm{m}^{-2} \mathrm{~s}^{-1}$, with the following order: $\mathrm{BR}<\mathrm{BP}<\mathrm{BA}<\mathrm{CK}$; average $\mathrm{CH}_{4}$ emissions (ME) ranged from 10.5 to $25.7 \mu \mathrm{mol} \mathrm{m}^{-2} \mathrm{~s}^{-1}$, with the following order: $\mathrm{CK}<\mathrm{BA}<\mathrm{BP}<\mathrm{BR}$. Average $\mathrm{CE}, \mathrm{NE}$ and $\mathrm{ME}$ were $36.5 \%$ lower in NT compared with $\mathrm{CT}$.

Microbial biomass $\mathrm{C}$ was positively correlated with total organic $\mathrm{C}(\mathrm{P}<0.05$, Table 5$)$ and negatively correlated with bulk density $(\mathrm{P}<0.01)$. Urease was positively correlated with $\mathrm{NO}_{3}-\mathrm{N}(\mathrm{P}<0.05) . \mathrm{CH}_{4}$ emissions were positively correlated with exchangeable $\mathrm{Mg}$ and macroaggregate $(\mathrm{P}<0.01)$.

\section{Banana yield}

Rotation effect was significant on banana yields $(\mathrm{P}<0.05$, Table 2$)$. The average banana yields in BA, BP and BR were, respectively, $41.6 \%, 58.7 \%$ and $49.9 \%$ higher than those in $\mathrm{CK}$, and $23.3 \%$ increase was observed in no-tillage compared with conventional tillage (Table 2). 
Table 4. Two-way ANOVA table of Pvalues showing the significance of the effects of different crop rotation and tillage practices on soil properties in a long-term crop rotation experiment.

\begin{tabular}{|c|c|c|c|c|c|c|c|}
\hline Indicators & Rotation (R) & Tillage (T) & Stage (S) & $\mathrm{R} \times \mathrm{T}$ & $\mathrm{R} \times \mathrm{S}$ & $\mathrm{T} \times \mathrm{S}$ & $\mathrm{R} \times \mathrm{T} \times \mathrm{S}$ \\
\hline SM & $<0.01$ & $<0.01$ & ns & $<0.01$ & 0.037 & ns & ns \\
\hline $\mathrm{BD}$ & $<0.01$ & $<0.01$ & $<0.01$ & ns & $<0.01$ & ns & ns \\
\hline $\mathrm{PO}$ & 0.044 & 0.019 & $<0.01$ & 0.043 & ns & 0.048 & ns \\
\hline $\mathrm{WSA}_{1}$ & $<0.01$ & $<0.01$ & ns & ns & ns & ns & 0.020 \\
\hline $\mathrm{WSA}_{2}$ & $<0.01$ & 0.031 & 0.049 & ns & ns & ns & 0.024 \\
\hline $\mathrm{pH}$ & $<0.01$ & $<0.01$ & ns & 0.018 & ns & ns & ns \\
\hline SOC & $<0.01$ & $<0.01$ & ns & $<0.01$ & ns & ns & ns \\
\hline $\mathrm{TN}$ & $<0.01$ & $<0.01$ & $<0.01$ & $<0.01$ & ns & ns & ns \\
\hline $\mathrm{NH}_{4}-\mathrm{N}$ & $<0.01$ & 0.049 & $<0.01$ & $\mathrm{~ns}$ & ns & ns & ns \\
\hline $\mathrm{NO}_{3}-\mathrm{N}$ & $<0.01$ & $<0.01$ & $<0.01$ & $<0.01$ & ns & ns & ns \\
\hline AP & $<0.01$ & $<0.01$ & ns & 0.016 & ns & ns & ns \\
\hline $\mathrm{AK}$ & 0.042 & $<0.01$ & $<0.01$ & 0.033 & $<0.01$ & ns & ns \\
\hline $\mathrm{ECa}$ & $<0.01$ & 0.039 & ns & ns & ns & ns & ns \\
\hline EMg & $<0.01$ & ns & ns & ns & ns & ns & ns \\
\hline $\mathrm{MBC}$ & $<0.01$ & $<0.01$ & $<0.01$ & ns & ns & ns & ns \\
\hline MBN & $<0.01$ & $<0.01$ & $<0.01$ & ns & 0.047 & ns & ns \\
\hline UA & $<0.01$ & $<0.01$ & $<0.01$ & ns & ns & ns & ns \\
\hline IA & $<0.01$ & $<0.01$ & $<0.01$ & 0.023 & ns & ns & ns \\
\hline DHA & $<0.01$ & $<0.01$ & $<0.01$ & ns & ns & ns & ns \\
\hline APA & $<0.01$ & 0.022 & ns & ns & ns & ns & ns \\
\hline GA & $<0.01$ & $<0.01$ & $<0.01$ & ns & $<0.01$ & ns & ns \\
\hline $\mathrm{CA}$ & $<0.01$ & $<0.01$ & $<0.01$ & ns & ns & ns & ns \\
\hline $\mathrm{NE}$ & 0.026 & 0.013 & 0.027 & ns & 0.014 & ns & ns \\
\hline $\mathrm{CE}$ & 0.017 & $<0.01$ & 0.031 & ns & ns & ns & ns \\
\hline $\mathrm{ME}$ & $<0.01$ & $<0.01$ & $<0.01$ & $<0.01$ & ns & ns & ns \\
\hline Yield & $<0.01$ & $<0.01$ & $<0.01$ & 0.029 & ns & ns & ns \\
\hline
\end{tabular}

SM: Soil moisture; BD: bulk density; PO: porosity; $\mathrm{WSA}_{1}$ : water-stable macroaggregate; WSA $\mathrm{W}_{2}$ : water-stable microaggregate; SOC: soil total organic C; TN: total N; AP: available P; AK: available K; ECa: exchangeable $\mathrm{Ca}$; EMg: exchangeable Mg; MBC: microbial biomass C; MBN: microbial biomass N; UA: urease activity; IA: invertase activity; DHA: dehydrogenase activity; APA: acid phosphatase activity; GA: $\beta$-glucosidase activity; CA: catalase activity; $\mathrm{NE}$ : $\mathrm{N}_{2} \mathrm{O}$ emission; $\mathrm{CE}$ : $\mathrm{CO}_{2}$ emission; $\mathrm{ME}$ : $\mathrm{CH}_{4}$ emission; ns: nonsignificant.

\section{DISCUSSION}

\section{Effects of rotation on soil quality}

$\mathrm{pH}$ increased 0.89 units in the rotations compared with monoculture, which is in agreement with the results of Dasa et al. (2018). These results suggest that root exudates of pineapple, cowpea or rice mitigate soil acidification. Total $\mathrm{N}$ and $\mathrm{NO}_{3}-\mathrm{N}$ were much higher in $\mathrm{BA}$ than in $\mathrm{CK}$, which were consistent with our previous studies (Zhong and Zeng, 2019). The differences were due to larger root quantity in rotations decreasing percolation water with $\mathrm{N}$ nutrients than in monoculture (Belmonte et al., 2018). AP and AK were on average $34.6 \%$ higher in BA and $43.7 \%$ higher in BP than in CK. Other researchers found similar results (Xu et al., 2020). They suggested that soil $\mathrm{pH}$ maintaining neutral range in rotations prevents $\mathrm{P}$ and $\mathrm{K}$ from fixation.

Urease, invertase, dehydrogenase and $\beta$-glucosidase obtained a mean of $51.7 \%$ increase in BP and $40.2 \%$ increase in BR compared with CK. These results were consistent with those of previous studies (Singh et al., 2018) that soil enzymes were stimulated by various crop sequences. The possible reason is that compared with monoculture plots, rotation plots have improved soil structure (Yang et al., 2020), stabilized microclimate (Adegaye et al., 2019) and greater abundance of rhizospheric microbes (mainly including rhizobia, gram-negative bacteria and Pseudomonas). Rotations emitted large amounts of $\mathrm{CH}_{4}$ to atmosphere, especially flooding rice cultivation. Pareja-Sánchez et al. (2019) reported similar findings. In their view, soil moisture and $\mathrm{Fe}^{2+}$ in $\mathrm{BR}$ were significantly higher than those of less irrigated treatments, thus increasing the abundance of methanogenesis. 
Figure 2. Soil microbial properties and enzyme activities in rotation and tillage treatments at four sampling stages in a long-term crop rotation experiment.


Error bars indicate coefficient of variation. Different letters mean significant difference according to Duncan's multiple range test $(\mathrm{P}<0.05)$. BA: Banana-pineapple rotation; BP: banana-cowpea rotation; BR: banana-rice rotation; CK: banana monoculture; NT: no-tillage; CT: conventional tillage; TPF: triphenylformazan. 
Figure 3. Greenhouse gas emissions in rotation and tillage treatments at eight sampling stages in a long-term crop rotation experiment.
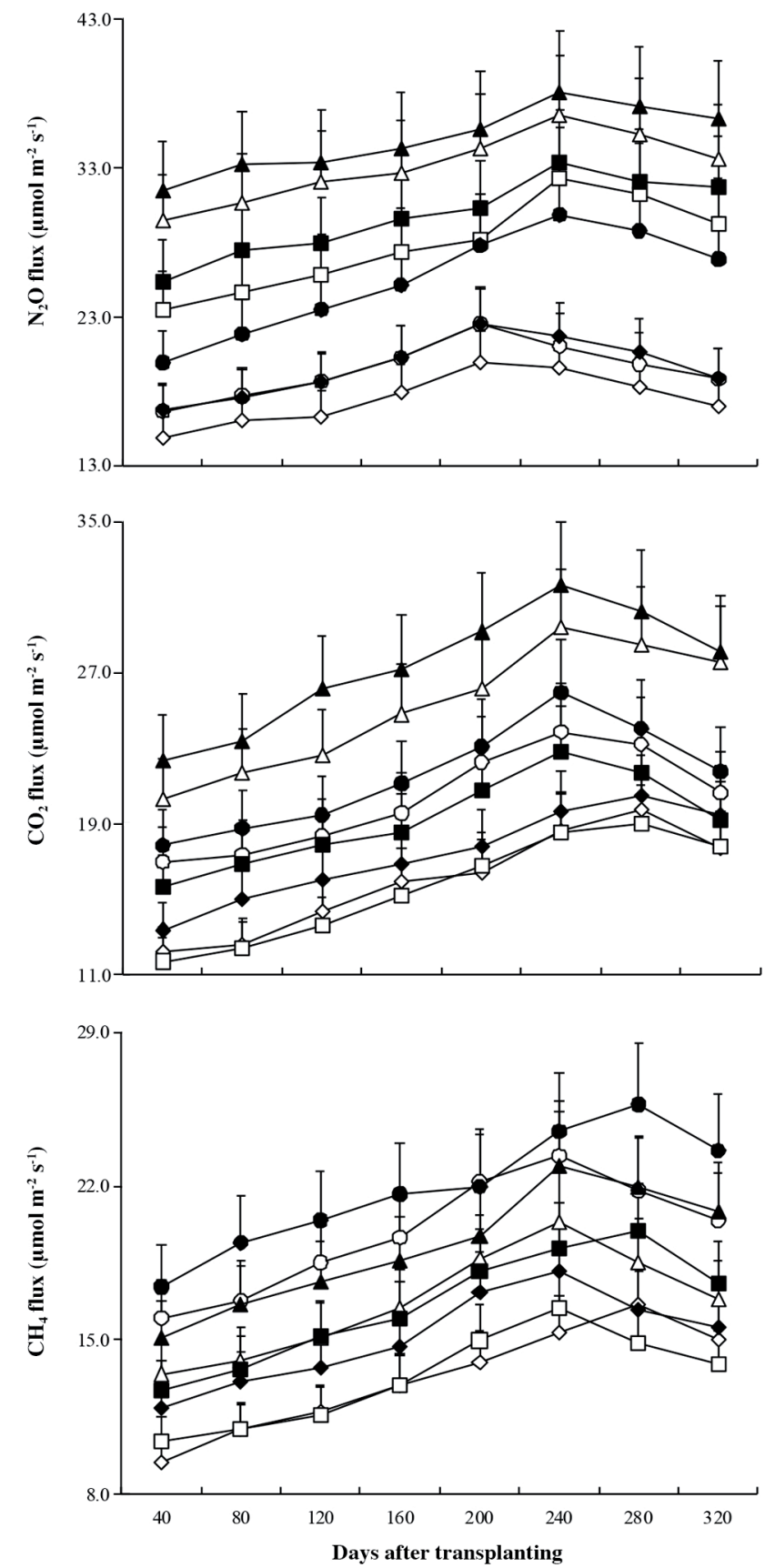

$\multimap$ CKCT $\neg$ ВACT $\neg$ BPCT $\multimap$ BRCT $\multimap$ CKNT $\rightarrow$ BANT $\neg$ BPNT $\bullet$ BRNT

Error bars indicate coefficient of variation. Different letters mean significant difference according to Duncan's multiple range test $(\mathrm{P}<0.05)$. BA: Banana-pineapple rotation; BP: banana-cowpea rotation; BR: banana-rice rotation; CK: banana monoculture; NT: no-tillage; CT: conventional tillage. 
Table 5. Pearson correlation coefficients between physicochemical-microbiological parameters and greenhouse gas across rotation and tillage treatments

\begin{tabular}{|c|c|c|c|c|c|c|c|c|c|c|c|}
\hline Indicators & $\mathrm{MBC}$ & MBN & UA & IA & GA & APA & DA & CA & $\mathrm{CE}$ & $\mathrm{NE}$ & $\mathrm{ME}$ \\
\hline SM & 0.307 & 0.047 & $0.614 * *$ & -0.277 & 0.209 & 0.362 & -0.323 & 0.156 & $-0.462 *$ & 0.142 & -0.293 \\
\hline $\mathrm{BD}$ & $-0.726^{* *} *$ & $-0.495 *$ & 0.223 & 0.059 & 0.120 & 0.178 & 0.446 & 0.197 & 0.334 & 0.301 & 0.395 \\
\hline PO & 0.174 & $0.566^{*}$ & $0.682 * *$ & 0.155 & 0.367 & $0.547 *$ & 0.434 & 0.228 & 0.057 & 0.076 & 0.099 \\
\hline $\mathrm{WSA}_{1}$ & 0.420 & 0.349 & 0.039 & $0.671 * *$ & 0.008 & -0.106 & $0.710 * *$ & 0.045 & $-0.580 *$ & 0.180 & $0.633 * *$ \\
\hline $\mathrm{WSA}_{2}$ & $0.538^{*}$ & 0.136 & 0.388 & -0.395 & $0.505^{*}$ & -0.475 & 0.179 & $0.553 *$ & $-0.528 *$ & 0.453 & 0.065 \\
\hline $\mathrm{pH}$ & 0.370 & 0.143 & $-0.568 *$ & 0.007 & 0.072 & $-0.606 * *$ & 0.133 & $0.653 * *$ & 0.231 & 0.036 & 0.250 \\
\hline SOC & $0.588 *$ & 0.367 & 0.249 & 0.040 & 0.029 & 0.168 & 0.061 & -0.196 & $0.504 *$ & 0.143 & $0.470^{*}$ \\
\hline $\mathrm{TN}$ & 0.137 & 0.416 & $0.507 *$ & 0.152 & 0.125 & $0.511 *$ & $0.575^{*}$ & 0.069 & 0.385 & 0.439 & 0.198 \\
\hline $\mathrm{NH}_{4}-\mathrm{N}$ & 0.030 & $0.670 * *$ & 0.319 & -0.049 & -0.178 & $0.596^{*}$ & 0.304 & 0.210 & 0.108 & $0.528^{*}$ & $0.467 *$ \\
\hline $\mathrm{NO}_{3}-\mathrm{N}$ & 0.296 & $0.479 *$ & $0.532 *$ & 0.285 & 0.303 & 0.424 & 0.407 & -0.273 & 0.263 & $0.602 * *$ & 0.274 \\
\hline $\mathrm{AP}$ & $0.544^{*}$ & 0.262 & $0.480 *$ & 0.134 & 0.311 & $0.463 *$ & 0.329 & 0.138 & -0.165 & 0.094 & 0.163 \\
\hline $\mathrm{AK}$ & $0.508 *$ & $0.519 *$ & 0.415 & 0.183 & 0.355 & 0.259 & 0.111 & 0.085 & 0.066 & 0.187 & 0.052 \\
\hline $\mathrm{ECa}$ & 0.403 & $0.488^{*}$ & 0.336 & 0.354 & $0.566^{*}$ & 0.284 & 0.153 & $0.742 * *$ & 0.193 & 0.208 & -0.030 \\
\hline $\mathrm{EMg}$ & 0.364 & 0.201 & 0.400 & 0.416 & $0.449 *$ & 0.376 & -0.427 & 0.328 & 0.124 & -0.146 & $0.681 * *$ \\
\hline
\end{tabular}

*,**Significant at the 0.05 and 0.01 probability levels, respectively.

MBC: Microbial biomass C; MBN: microbial biomass N; UA: urease activity; IA: invertase activity; DHA: dehydrogenase activity; APA: acid

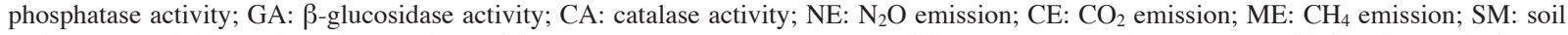
moisture; BD: bulk density; PO: porosity; $\mathrm{WSA}_{1}$ : water-stable macroaggregate; $\mathrm{WSA}_{2}$ : water-stable microaggregate; $\mathrm{SOC}$ : soil organic C; TN: total N; AP: available P; AK: available K; ECa: exchangeable $\mathrm{Ca}$; $\mathrm{EMg}$ : exchangeable $\mathrm{Mg}$.

\section{Effects of tillage on soil quality}

Soil moisture and water-stable aggregates increased on average $32.5 \%$ in NT compared with CT, which were because crop residues returning on soil surface reduced evaporation and less soil disturbance improved soil structure. The results were in agreement with other studies (Zhang et al., 2017). In NT plots, soil consolidation will continue augment until an equilibrium bulk density is reached, range of $1.2-1.5 \mathrm{~g} \mathrm{~cm}^{-3}$ for our soil type (Sayed et al., 2019). Our results were consistent with those of Sauvadet et al. (2018), who found that reduced tillage doubled soil organic C (SOC) compared with CT. They suggested that residues covering on soil surface decreased soil temperature and prevented $\mathrm{C}$ mineralization. The content of exchangeable Ca (ECa) in NT was $35.7 \%$ higher than that in conventional tillage. Similar results were reported in same soil types (He et al., 2020b). The process was explained as crop residues in NT released large amount of soluble $\mathrm{Ca}^{2+}$ to replace $\mathrm{H}^{+}$(Moghimian et al., 2019).

Average MBC and MBN increased $40.3 \%$ and $32.5 \%$ in NT compared with CT. The positive responses of microbial biomass to NT were attributed to the large amount of $\mathrm{C}$ and $\mathrm{N}$ substrates provided by cowpea and pineapple residues addition (Saikia et al., 2019). $\mathrm{N}_{2} \mathrm{O}$ and $\mathrm{CO}_{2}$ emissions were $32.5 \%$ and $22.9 \%$ lower in NT than in CT. The results were consistent with the studies of Behnke et al. (2018). Unlike CT, which distributes organic matter in 0-40 cm plow layer, NT leaves organic matter close to soil surface, thus, it prevents the release of greenhouse gases into the atmosphere.

\section{Effects of stage on soil quality}

Microbial biomass C, acid phosphatase and dehydrogenase gradually increased and reached peak at booting then decreased afterwards. The fluctuation was consistent with banana growth. Notaro et al. (2018) also observed an increase of rhizosphere products, such as root exudates, mucilage and sloughed cells at booting, which increase the abundance of rhizospheric microbes.

$\mathrm{N}_{2} \mathrm{O}$ and $\mathrm{CO}_{2}$ emissions increased dramatically from March to May, which were the warmest season of 2019-2020. Similar results were reported by Piotrowska-Dlugosz et al. (2019). They attributed the reasons to the continuous increase of soil moisture, temperature and $\mathrm{C}$ input from jointing to booting.

\section{CONCLUSIONS}

The increased soil microbial biomass and enzyme activities in rotation soils are accompanied by an increase in microbial activity. The decreased greenhouse gas emissions and increased soil organic $\mathrm{C}$ and total $\mathrm{N}$ could explain the higher $\mathrm{C}$ and $\mathrm{N}$ sequestration in no-tillage than in conventional tillage. Therefore, rotation and no-tillage practices effectively improved 
soil quality and increased banana yield in tropical environments. In conclusion, integration of no-tillage into rotation systems provides a sustainable management in banana production.

\section{ACKNOWLEDGEMENTS}

This research was supported by the National Natural Science Foundation of China (41301277), Central Public-interest Scientific Institution Basal Research Fund for Chinese Academy of Tropical Agricultural Sciences (1630092017005, 1630092017007), Key Research and Development Projects of Hainan Province (ZDYF2019187) and Hainan Provincial Natural Science Fund Project (319QN267).

\section{REFERENCES}

Adegaye, A., Adejoro, S., Oladele, S., Arotupin, D., and Ojeniyi, S. 2019. Short-term impacts of management practices on soil properties under maize cultivation in Akure, Ondo State, Nigeria. Scientific African 5:e00129.

Behnke, G.D., Zuber, S.M., Pittelkow, C.M., Nafziger, E.D., and Villamil, M.B. 2018. Long-term crop rotation and tillage effects on soil greenhouse gas emissions and crop production in Illinois, USA. Agriculture, Ecosystems and Environment 261:62-70.

Belmonte, S.A., Celi, L., Stahel, R.J., Bonifacio, E., Novello, V., Zanini, E., et al. 2018. Effect of long-term soil management on the mutual interaction among soil organic matter, microbial activity and aggregate stability in a vineyard. Pedosphere 28:288-298.

Bera, T., Sharma, S., Thind, H.S., Singh, Y., Sidhu, H.S., and Jat, M.L. 2018. Changes in soil biochemical indicators at different wheat growth stages under conservation-based sustainable intensification of rice-wheat system. Journal of Integrative Agriculture 17:1871-1880.

Brandan, C.P., Chavarría, D., Huidobro, J., Meriles, J.M., and Gil, S.V. 2017. Influence of a tropical grass (Brachiaria brizantha cv. Mulato) as cover crop on soil biochemical properties in a degraded agricultural soil. European Journal of Soil Biology 83:84-90

da Vitória, Y.T., Leite, M.C.T., Delgado, R.C., Moreira, G.F., Oliveira, E.C. de, Quartezani, W.Z., et al. 2019. Soil carbon dioxide efflux in Conilon coffee (Coffea canephora Pierre ex Froehner) plantations in different phenological phases in tropical climate in Brazil. Chilean Journal of Agricultural Research 79:366-375.

Dasa, A., Lyngdoh, D., Ghosh, P.K., Lal, R., Layek, J., and Idapuganti, R.G. 2018. Tillage and cropping sequence effect on physico-chemical and biological properties of soil in Eastern Himalayas, India. Soil and Tillage Research 180:182-193.

Deuschle, D., Minella, J.P.G., Hörbe, T.A.N., Londero, A.L., and Schneider, F.J.A. 2019. Erosion and hydrological response in no-tillage subjected to crop rotation intensification in southern Brazil. Geoderma 340:157-163.

He, H.B., Li, W.X., Zhang, Y.W., Cheng, J.K., Jia, X.Y., Li, S., et al. 2020a. Effects of Italian ryegrass residues as green manure on soil properties and bacterial communities under an Italian ryegrass (Lolium multiflorum L.)-rice (Oryza sativa L.) rotation. Soil and Tillage Research 196:104487.

He, L.Y., Zhang, A.F., Wang, X.D., Li, J., and Hussain, Q. 2019. Effects of different tillage practices on the carbon footprint of wheat and maize production in the Loess Plateau of China. Journal of Cleaner Production 234:297-305.

He, L.L., Zhao, J., Yang, S.M., Zhou, H., Wang, S.Q., Zhao, X., et al. 2020b. Successive biochar amendment improves soil productivity and aggregate microstructure of a red soil in a five-year wheat-millet rotation pot trial. Geoderma 376:114570.

Hulugalle, N.R., Weaver, T.B., Finlay, L.A., Hare, J., and Entwistle, P.C. 2007. Soil properties and crop yields in a dryland Vertisol sown with cotton-based crop rotations. Soil and Tillage Research 93:356-369.

Kubar, K.A., Li, H., Lu, J.W., Li, X.K., Xue, B., and Yin, Z.Y. 2018. Integrative effects of no-tillage and straw returning on soil organic carbon and water stable aggregation under rice-rape rotation. Chilean Journal of Agricultural Research 78:205-215.

Legrand, F., Picot, A., Cobo-Díaz, J.F., Carof, M., Chen, W., and Le Floch, G. 2018. Effect of tillage and static abiotic soil properties on microbial diversity. Applied Soil Ecology 132:135-145.

Moghimian, N., Hosseini, S.M., Kooch, Y., and Darki, B.Z. 2019. Evaluating soil biochemical/microbial indices as ecological indicators of different land use/cover in northern Iran. Acta Ecologica Sinica 39:328-333.

Mu, X.Y., Zhao, Y.L., Liu, K., Ji, B.Y., Guo, H.B., Xue, Z.W., et al. 2016. Responses of soil properties, root growth and crop yield to tillage and crop residue management in a wheat-maize cropping system on the North China Plain. European Journal of Agronomy 78:32-43.

Notaro, K.A., de Medeiros, E.V., Duda, G.P., Moreira, K.A., de Barros, J.A., dos Santos, U.J., et al. 2018. Enzymatic activity, microbial biomass, and organic carbon of Entisols from Brazilian tropical dry forest and annual and perennial crops. Chilean Journal of Agricultural Research 78:68-77.

Pareja-Sánchez, E., Cantero-Martínez, C., Álvaro-Fuentes, J., and Plaza-Bonilla, D. 2019. Tillage and nitrogen fertilization in irrigated maize: key practices to reduce soil $\mathrm{CO}_{2}$ and $\mathrm{CH}_{4}$ emissions. Soil and Tillage Research 191:29-36. 
Piotrowska-Dlugosz, A., Breza-Boruta, B., and Dlugosz, J. 2019. Spatial and temporal variability of the soil microbiological properties in two soils with a different pedogenesis cropped to winter rape (Brassica napus L.) Geoderma 340:313-324.

Saikia, R., Sharma, S., Thind, H.S., Sidhu, H.S., and Singh, Y. 2019. Temporal changes in biochemical indicators of soil quality in response to tillage, crop residue and green manure management in a rice-wheat system. Ecological Indicators 103:383-394.

Sauvadet, M., Lashermes, G., Alavoine, G., Recous, S., Chauvat, M., Maron, P., et al. 2018. High carbon use efficiency and low priming effect promote soil C stabilization under reduced tillage. Soil Biology and Biochemistry 123:64-73.

Sayed, A., Sarker, A., Kim, J.E., Rahman, M., and Mahmud, G.A. 2019. Environmental sustainability and water productivity on conservation tillage of irrigated maize in red brown terrace soil of Bangladesh. Journal of the Saudi Society of Agricultural Sciences 19(4):276-284.

Shiwakoti, S., Zheljazkov, V.D., Gollany, H.T., Kleber, M., and Xing, B.S. 2019. Effect of tillage on macronutrients in soil and wheat of a long-term dryland wheat-pea rotation. Soil and Tillage Research 190:194-201.

Singh, G., Bhattacharyya, R., Das, T.K., Sharma, A.R., Ghosh, A., Das, S., et al. 2018. Crop rotation and residue management effects on soil enzyme activities, glomalin and aggregate stability under zero tillage in the Indo-Gangetic Plains. Soil and Tillage Research 184:291-300.

Tabatabai, A. 1994. Soil enzymes. p. 775-833. In Weaver, R.W., Angle, J.S., and Bottomley, P.S. (eds.) Methods of soil analysis. Part 2. Microbiological and biological properties. $2^{\text {nd }}$ ed. Soil Science Society of America, Madison, Wisconsin, USA.

Vance, E.D., Brookes, P.C., and Jenkinson, D.S. 1987. An extraction method for measuring microbial biomass C. Soil Biology and Biochemistry 19:703-707.

Wairegi, L.W.I., and van Asten, P.J.A. 2010. The agronomic and economic benefits of fertilizer and mulch use in highland banana systems in Uganda. Agricultural Systems 108:543-550.

Xavier, C.V., Moitinho, M.R., Teixeira, D.B., Santos, G.A., Barbosa, M.A., Milori, M.B.P., et al. 2019. Crop rotation and succession in a no-tillage system: Implications for $\mathrm{CO}_{2}$ emission and soil attributes. Journal of Environmental Management 245:8-15.

Xu, Y.X., Du, A.P., Wang, Z.C., Zhu, W.K., Li, C., and Wu, L.C. 2020. Effects of different rotation periods of Eucalyptus plantations on soil physiochemical properties, enzyme activities, microbial biomass and microbial community structure and diversity. Forest Ecology and Management 456:117683.

Yang, H.S., Li, Y.F., Zhai, S.L., Fang, C., Liu, J., and Zhang, Q. 2020. Long term ditch-buried straw return affects soil fungal community structure and carbon-degrading enzymatic activities in a rice-wheat rotation system. Applied Soil Ecology 155:103660.

Yang, X., Zheng, L.N., Yang, Q., Wang, Z.K., Cui, S., and Shen, Y.Y. 2018. Modelling the effects of conservation tillage on crop water productivity, soil water dynamics and evapotranspiration of a maize-winter wheat-soybean rotation system on the Loess Plateau of China using APSIM. Agricultural Systems 166:111-123.

Zhang, X.F., Xin, X.L., Zhu, A.N., Zhang, J.B., and Yang, W.L. 2017. Effects of tillage and residue managements on organic C accumulation and soil aggregation in a sandy loam soil of the North China Plain. Catena 156:176-183.

Zhong, S., and Zeng, H.C. 2019. Influence of long-term tillage and residue management on soil biota in tropical climate. Journal of Biobased Materials and Bioenergy 13:576-584.

Zhong, S., and Zeng, H.C. 2020. Long-term interactions of reduced tillage and different amounts of residue retaining improved soil environment in a semi-arid tropical climate. Chilean Journal of Agricultural Research 80:197-208.

Zuber, S.M., Behnke, G.D., Nafziger, E.D., and Villamil, M.B. 2017. Multivariate assessment of soil quality indicators for crop rotation and tillage in Illinois. Soil and Tillage Research 174:147-155. 